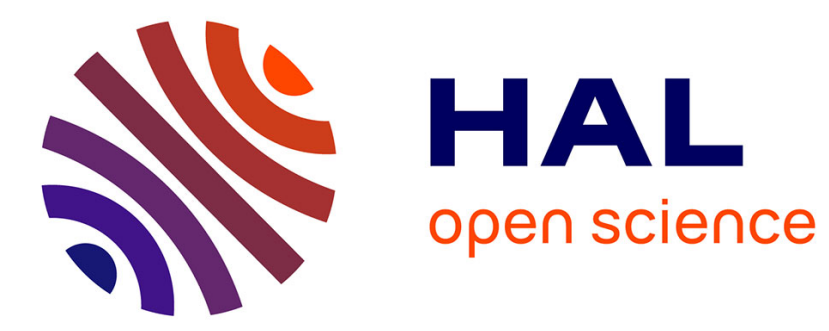

\title{
On arithmetical functions having constant values in some domain
}

Jay Mehta

\section{To cite this version:}

Jay Mehta. On arithmetical functions having constant values in some domain. Acta Mathematica Hungarica, 2013, 142, pp.110 - 117. 10.1007/s10474-013-0342-8 . hal-03238314

\section{HAL Id: hal-03238314 https://hal.science/hal-03238314}

Submitted on 1 Aug 2021

HAL is a multi-disciplinary open access archive for the deposit and dissemination of scientific research documents, whether they are published or not. The documents may come from teaching and research institutions in France or abroad, or from public or private research centers.
L'archive ouverte pluridisciplinaire HAL, est destinée au dépôt et à la diffusion de documents scientifiques de niveau recherche, publiés ou non, émanant des établissements d'enseignement et de recherche français ou étrangers, des laboratoires publics ou privés. 


\title{
ON ARITHMETICAL FUNCTIONS HAVING CONSTANT VALUES IN SOME DOMAIN
}

\author{
JAY MEHTA
}

\begin{abstract}
Dedicated to Professor Imre Kátai on the occasion of his 75th birthday.
\end{abstract}

\begin{abstract}
This paper shows that any completely additive complex valued function over a principal configuration in complex plane, having constant values in some discs is identically zero function. In other words, there exists no non-trivial completely additive complex valued function over a principal configuration in $\mathbb{C}$ which assumes constant values in some domain.
\end{abstract}

\section{INTRODUCTION}

Paul Erdős initiated the study of arithmetical functions with certain properties. He studied (completely) additive and multiplicative functions satisfying some specific properties. I. Kátai also studied such additive and multiplicative functions and has contributed in the same area (for example [5], [6], etc.). I. Kátai together with Paul Erdős has proved many interesting results in this field (for example see [3], [4], etc.). In this paper, we talk about one such example of completely additive and completely multiplicative function having constant values in some domain in the complex plane.

In 1969, I. Kátai proved in [5] that any completely additive real valued function on the set of positive integers assuming constant values in some intervals of real line is identically zero function. In 1991, M. Amer generalized this result for complex valued functions on the set of non-zero Gaussian integers. Amer proved that a completely additive complex valued functions defined on non-zero Gaussian integers having constant values in some discs of the complex plane is identically zero (Theorem 1 in [1]).

The aim is to generalize the result of M. Amer for completely additive complex valued functions over non-zero lattice points in complex plane. In general an arbitrary lattice (say $\Lambda_{1}$ ) may not be closed under

2010 Mathematics Subject Classification. 11A25,11N64.

Key words and phrases. multiplicative functions, Gaussian integers, additive functions, lattices, principal configuration. 
multiplication. The proof of the result proved here uses the 'closed under multiplication' property of the system of lattice points which may not be the case always. So in order to make our domain closed under multiplication we supplement the lattice $\Lambda_{1}$ with some additional points in such a way that this larger collection of points (which may no longer be a lattice), is still discrete and is closed under multiplication. Such a system of points is called 'principal configuration' (see [2], page 89-90). These additional points form finitely many lattices $\Lambda_{2}, \Lambda_{3}, \ldots \Lambda_{h}$ having only the origin $O$ in common with the lattice $\Lambda_{1}$ and with each other lattices. Let us denote the principal configuration by $\Gamma$. Then we have,

$$
\Gamma=\Lambda_{1} \cup \Lambda_{2} \cup \ldots \cup \Lambda_{h}
$$

Though each lattices $\Lambda_{i}$ are closed under addition, $\Gamma$ may not be closed under addition but it is closed under multiplication. The supplementary points (i.e. the points of $\Lambda_{i}, i=2,3, \ldots, h$ ) are called auxiliary or non-principal. Also, the fundamental parallelepiped of these lattices have the same complex volume as $\Lambda_{1}$. The geometric picture of above described situation in 2-dimensional case was first given by Klein in 1896 but it can be considered in any dimension.

We consider completely additive complex valued functions over nonzero points of the principal configuration $\Gamma$ and prove our result (see Theorem 1 in Section 2) which generalizes the result of M. Amer. A similar result for completely multiplicative functions, can be proved in the same way, is stated at the end of this paper (see Theorem 2).

In case the given lattice $\Lambda_{1}$ is already closed under multiplication, we have $h=1$ and our principal configuration $\Gamma$ will be the lattice $\Lambda_{1}$ itself. Thus, as a particular case of our result, we have the result of M. Amer as the set of non-zero Gaussian integers is closed under multiplication. As another corollary, one can consider the case of arithmetical functions over the ring of integers of imaginary quadratic fields.

\section{Preliminaries and the Result}

Let $\Gamma$ be the principal configuration and let $\Lambda_{i}=\Lambda_{i}\left(\omega_{i}, \omega_{i}^{\prime}\right)$ where $\omega_{i}, \omega_{i}^{\prime} \in \mathbb{C}, i=1,2, \ldots, h$ be the lattices in the principal configuration $\Gamma$. That is we have

$$
\Gamma=\Lambda_{1} \cup \Lambda_{2} \cup \ldots \cup \Lambda_{h}
$$

Throughout this paper, without loss of generality, we assume that $\left|\omega_{i}^{\prime}\right| \leq\left|\omega_{i}\right|$ for all $i=1,2, \ldots h$. Let $\Gamma^{*}$ denote the set of all nonzero points of $\Gamma$. We denote the set of all completely additive functions and completely multiplicative complex valued functions over $\Gamma^{*}$ by $\mathcal{A}_{\Gamma}^{*}$ 
and $\mathcal{M}_{\Gamma}^{*}$ respectively. Let $S(a, r)(\subseteq \mathbb{C})$ be the closed disc with center $a$ and radius $r$, i.e.,

$$
S(a, r)=\{z \in \mathbb{C}|| z-a \mid \leq r\} .
$$

Let $\omega^{\prime}=\omega_{j}^{\prime}$ and $\omega=\omega_{k}$ for some $j$ and $k$ such that

$$
\left|\omega^{\prime}\right|=\left|\omega_{j}^{\prime}\right|=\min _{1 \leq i \leq h}\left\{\left|\omega_{i}^{\prime}\right|\right\} ; \quad|\omega|=\left|\omega_{k}\right|=\max _{1 \leq i \leq h}\left\{\left|\omega_{i}\right|\right\} .
$$

i.e., $\omega^{\prime}$ is one of the $\omega_{i}^{\prime \prime}$ s which has the minimum absolute value while $\omega$ is one of the $\omega_{i}^{\prime}$ s which has maximum absolute value. We keep the same notation throughout the paper.

We prove the following:

Theorem 1. Let $f \in \mathcal{A}_{\Gamma}^{*}$. Assume that there exists a sequence of complex numbers $z_{1}, z_{2}, \ldots$ such that $\left|z_{j}\right| \rightarrow \infty(j \rightarrow \infty)$ and that

$$
f(\alpha)=A_{j} \text { (constant) for all } \alpha \in S\left(z_{j},(2+\epsilon) \sqrt{\left|z_{j}\right|}\right)
$$

for some positive constant $\epsilon$ depending on the principal configuration $\Gamma$. Then $f \equiv 0$.

\section{Proof of TheOrem 1}

Theorem 1 follows easily from the following three lemmas:

Lemma 1. Let $f \in \mathcal{A}_{\Gamma}^{*}, z \in \Gamma^{*}$ with $|z|=M\left(\geq\left|\omega_{j}\right|+\left|\omega_{j}^{\prime}\right|\right)$. Assume that $f(\alpha)=A$ (constant) in the annulus $R=\left\{\alpha \in \Gamma^{*}|M \leq| \alpha \mid \leq\right.$ $\left.\left|\omega_{j}+\omega_{j}^{\prime}\right| M\right\}$. Then $f$ vanishes in the whole disc with radius same as the outer radius of the annulus $R$. i.e., $f(\alpha)=0$ for every $\alpha \in \Gamma^{*}$ with $|\alpha| \leq\left|\omega_{j}+\omega_{j}^{\prime}\right| M$.

NOTE: In Lemma 1, the construction of our annulus $R$ depends on $\omega_{j}$ and $\omega_{j}^{\prime}$ of the lattice $\Lambda_{j}\left(\omega_{j}^{\prime}, \omega_{j}\right)$. Note that, we can use any lattice $\Lambda_{i}$ of $\Gamma$ in the construction of $R$ but we have used $\Lambda_{j}$ as $\omega_{j}^{\prime}$ has the minimum absolute value and we use this fact in the proof of Lemma 2 on page 6 line number 8 .

Proof. Let $\lambda=\omega_{j}+\omega_{j}^{\prime} \in \Lambda_{j}$. Then $|\lambda z|=|\lambda||z|=\left|\omega_{j}+\omega_{j}^{\prime}\right| M$. Therefore, $z, \lambda z \in R$. As $f$ is completely additive function, we have

$$
f(\lambda)=f(\lambda z)-f(z)=0 .
$$

Let $k \in \mathbb{N}$ such that $\lambda^{k} \in R$. It is clear from the construction of $R$ that such $k$ exists. Then

$$
A=f\left(\lambda^{k}\right)=k f(\lambda)=0 .
$$


Now, let $\alpha \in \Gamma^{*}$ with $|\alpha|<M$. Choose suitable $m \in \mathbb{N}$ such that $\alpha \lambda^{m} \in R$. Consequently,

$$
0=A=f\left(\alpha \lambda^{m}\right)=f(\alpha)+m f(\lambda)=f(\alpha) .
$$

Hence the lemma.

Let

$$
[r]_{\Gamma}=\max _{\alpha \in \Gamma^{*},|\alpha| \leq r}|\alpha|,
$$

for $r \in \mathbb{R},(r>1)$. It is clear that, $[r]_{\Gamma} \leq r$.

Lemma 2. Let $\delta$ be an arbitrary number such that $0<\delta<1$. Then there exists a constant $N_{0}$, depending on $\delta$ and $\Gamma$, with the following property:

If $f \in \mathcal{A}_{\Gamma}^{*}$ and $N \in \mathbb{R}, N>N_{0}, f(\alpha)=A$ for $\alpha \in R_{N}=\{\alpha \in$ $\left.\Gamma^{*}|N \leq| \alpha \mid \leq(1+\delta) N\right\}$, then $f(\alpha)=0$ for each $\alpha \in \Gamma^{*}$ with $|\alpha| \leq\left[\frac{\delta N}{2\left|\omega^{\prime}\right|}-|\omega|\right]_{\Gamma}$.

Proof. Let $\beta \in \Gamma^{*},|\beta|<N$. Then $\beta \in \Lambda_{i}^{*} \subset \Gamma^{*}$ for some $i$. Let $E_{i}=\left\{ \pm \omega_{i}, \pm \omega_{i}^{\prime}, 0\right\}$ and $\theta \in E_{i}$. Suppose

$$
\frac{N(1+\delta)}{|\beta|+|\omega|}-\frac{N}{|\beta|-|\omega|} \geq\left|\omega^{\prime}\right|
$$

holds.

Our aim is to show that $f$ is constant for all $\beta \in \Gamma^{*}$ for which (1) holds. For this we will show that $f$ is constant for all $\beta \in \Lambda_{i}^{*}$ for which (1) holds for all $i=1, \ldots, h$.

Suppose

$$
\frac{N(1+\delta)}{|\beta|+\left|\omega_{i}\right|}-\frac{N}{|\beta|-\left|\omega_{i}\right|} \geq\left|\omega_{i}^{\prime}\right|
$$

holds. Then there exists some $\mu \in \Gamma^{*}$ such that

$$
\frac{N}{|\beta|-\left|\omega_{i}\right|} \leq|\mu| \leq \frac{N(1+\delta)}{|\beta|+\left|\omega_{i}\right|} \text {. }
$$

But then

and so

$$
\frac{N}{|\beta+\theta|} \leq|\mu| \leq \frac{N(1+\delta)}{|\beta+\theta|}
$$

$$
N \leq|(\beta+\theta) \mu| \leq N(1+\delta)
$$

This implies $(\beta+\theta) \mu \in R_{N}$. Since $\theta=0 \in E$ we have $A=f(\mu \beta)=$ $f((\beta+\theta) \mu)$. As $f$ is completely additive function, we have

$$
f(\beta)=f(\beta+\theta) .
$$


This is true for all $i=1,2, \ldots, h$ provided that equation (A) holds for all $i$. Clearly, (by definition of $\omega$ and $\omega^{\prime}$ ) we have,

$$
\frac{N(1+\delta)}{|\beta|+\left|\omega_{i}\right|}-\frac{N}{|\beta|-\left|\omega_{i}\right|} \geq \frac{N(1+\delta)}{|\beta|+|\omega|}-\frac{N}{|\beta|-|\omega|} \geq\left|\omega^{\prime}\right| \geq\left|\omega_{i}^{\prime}\right|
$$

Hence, we can say that equation (A) holds for all $i$ if (1) holds. Thus, we have proved that $f$ is constant for all such $\beta \in \Gamma^{*}$ for which (1) holds.

Now we determine for what $\beta$, equation (1) holds. Equation (1) implies

$$
\left|\omega^{\prime}\right||\beta|^{2}-N \delta|\beta|+N \delta|\omega|+2 N|\omega|-\left|\omega^{\prime}\right||\omega|^{2} \leq 0
$$

We shall prove that inequality (1) holds for $\beta$ if

$$
|\beta| \in L:=\left[|\omega|\left(\frac{4}{\delta}+1\right), \frac{\delta N}{2\left|\omega^{\prime}\right|}-|\omega|\right] .
$$

As (2) is a quadratic equation in $|\beta|$, it suffices to show that (1) holds for the end-points on the interval $L$.

First let $|\beta|=|\omega|\left(\frac{4}{\delta}+1\right)$. Substituting this value of $|\beta|$ in (1), the left hand side of (1) will be

$$
\frac{\delta^{2} N}{|\omega|(4 \delta+8)}
$$

which is clearly $\geq\left|\omega^{\prime}\right|$ if

$$
N \geq|\omega|\left|\omega^{\prime}\right|\left(\frac{4}{\delta}+\frac{8}{\delta^{2}}\right)
$$

Now, let $|\beta|=\frac{\delta N}{2\left|\omega^{\prime}\right|}-|\omega|$. Substituting this value of $|\beta|$ in (1), the left hand side of (1) will be

$$
\frac{2 \delta^{2} N\left|\omega^{\prime}\right|-8 \delta|\omega|\left|\omega^{\prime}\right|^{2}-8|\omega|\left|\omega^{\prime}\right|^{2}}{\delta^{2} N-4 \delta|\omega|\left|\omega^{\prime}\right|}
$$

and this is $\geq\left|\omega^{\prime}\right|$ if

$$
N \geq|\omega|\left|\omega^{\prime}\right|\left(\frac{4}{\delta}+\frac{8}{\delta^{2}}\right)
$$

Let

$$
N_{0}=|\omega|\left|\omega^{\prime}\right|\left(\frac{4}{\delta}+\frac{8}{\delta^{2}}\right)
$$

be the constant. We have proved that $f(\beta)=f(\beta+\theta)$, whenever $|\beta| \in L, \beta \in \Gamma^{*}$ and $N \geq N_{0}$. This means that $f$ is constant in the annulus $|\beta| \in L$. Now, clearly we have

$$
\left[\frac{\delta N}{2\left|\omega^{\prime}\right|}-|\omega|\right]_{\Gamma} \geq|\omega|+\left|\omega^{\prime}\right|\left[|\omega|\left(\frac{4}{\delta}+1\right)\right]_{\Gamma}
$$


for sufficiently large $N$ (as right hand side contains $N$ and left hand side is independent of $N)$. Then we can find some $z \in \Gamma^{*}$ such that

$$
\left(|z|,\left(|\omega|+\left|\omega^{\prime}\right|\right)|z|\right) \subseteq L .
$$

But clearly,

$$
\left(|z|,\left|\omega_{j}+\omega_{j}^{\prime}\right||z|\right) \subseteq\left(|z|,\left(\left|\omega_{j}\right|+\left|\omega_{j}^{\prime}\right|\right)|z|\right) \subseteq\left(|z|,\left(|\omega|+\left|\omega^{\prime}\right|\right)|z|\right)
$$

as $\left|\omega_{j}^{\prime}\right|=\left|\omega^{\prime}\right|$ and $\left|\omega_{j}\right| \leq|\omega|$.

So we have,

$$
\left(|z|,\left|\omega_{j}+\omega_{j}^{\prime}\right||z|\right) \subseteq\left(|z|,\left(|\omega|+\left|\omega^{\prime}\right|\right)|z|\right) \subseteq L .
$$

Applying Lemma 1 for $|z|$ in place of $M$, we get $f(\alpha)=0$ whenever $|\alpha| \leq\left(|\omega|+\left|\omega^{\prime}\right|\right)|z|$ and hence for $|\alpha| \leq\left|\omega_{j}+\omega_{j}^{\prime}\right||z|$. But $f(\alpha)$ is constant whenever $|\alpha| \in L$ and hence $f(\alpha)=0$ for the whole disc stated in the lemma 2.

Lemma 3. Let $\epsilon>0$ be some fixed constant depending on $\Gamma$. Then there exists positive numbers $N_{1}$ and $c$, depending on $\epsilon$ and the principal configuration $\Gamma$, with the following properties:

If $f \in \mathcal{A}_{\Gamma}^{*}, a \in \mathbb{C}$ satisfying $|a|>N_{1}, r=(2+\epsilon) \sqrt{|a|}$ and $f(\alpha)=A$ (constant) in the disc $S(a, r)$, then $f(\alpha)=0$ for each $\alpha \in \Gamma^{*}$ such that $|\alpha| \leq c \sqrt{|a|}$.

Proof. For each $\alpha \in \mathbb{C}$, the disc $S(\alpha,|\omega|)$ contains at least one element of $\Gamma$. Let $\beta \in \Gamma^{*}$ (say $\beta \in \Lambda_{i}^{*}$ for some $i$ ) with $|\beta| \leq \frac{r}{|\omega|}$. Then there exists some $\mu \in \Gamma^{*}$ such that

$$
\left|\mu-\frac{a}{\beta}\right| \leq|\omega| \leq \frac{r}{|\beta|} .
$$

Let $E_{i}=\left\{ \pm \omega_{i}, \pm \omega_{i}^{\prime}\right\}$ and $\theta \in E_{i}$ and assume that $\beta$ is so chosen that the following equation holds:

$$
|\omega|+\frac{|a||\theta|}{|\beta||\beta+\theta|} \leq \frac{r}{|\beta+\theta|} .
$$

Then

$$
\begin{aligned}
\left|\mu-\frac{a}{\beta+\theta}\right| & \leq\left|\mu-\frac{a}{\beta}\right|+\left|\frac{a}{\beta}-\frac{a}{\beta+\theta}\right| \\
& \leq|\omega|+\frac{|a||\theta|}{|\beta||\beta+\theta|} \leq \frac{r}{|\beta+\theta|}
\end{aligned}
$$

As a consequence of (3) and (5), we have $\mu \beta$ and $\mu(\beta+\theta) \in S(a, r)$. By hypothesis, we have $f(\mu \beta)=A=f(\mu(\beta+\theta))$. Now, as $f$ is completely additive function, we have $f(\beta)=f(\beta+\theta)$, provided that (4) holds. 
This is true for all $i=1,2, \ldots, h$. Thus, we have proved that $f$ is constant for all $\beta \in \Gamma^{*}$ for which (4) holds.

By simple computation, we see that (4) holds for each $\theta \in E_{i}$ for each $i$ if

$$
|\beta||\omega|(|\beta|+|\omega|)-r|\beta|+|a||\omega| \leq 0
$$

The above inequality holds in the interval $|\beta| \in\left(x_{1}, x_{2}\right)$ where $x_{1}$ and $x_{2}$ are the roots of the following quadratic equation:

$$
|\omega| x^{2}-\left(r-|\omega|^{2}\right) x+|a||\omega|=0
$$

where $|a|$ is sufficiently large and

$$
\epsilon \geq 2(|\omega|-1) .
$$

Then,

and

$$
x_{1}=\frac{\left(r-|\omega|^{2}\right)-\sqrt{\left(r-|\omega|^{2}\right)^{2}-4|a||\omega|^{2}}}{2|\omega|}
$$

$$
x_{2}=\frac{\left(r-|\omega|^{2}\right)+\sqrt{\left(r-|\omega|^{2}\right)^{2}-4|a||\omega|^{2}}}{2|\omega|} .
$$

We note that $x_{1}(|a|) \rightarrow \infty$ as $|a| \rightarrow \infty$ and that

$$
\begin{aligned}
\frac{2|\omega| x_{1}}{r} & =1-\sqrt{\left(1-\frac{|\omega|^{2}}{r}\right)^{2}-\frac{4|a||\omega|^{2}}{r^{2}}}-\frac{|\omega|}{r} \\
& =1-\sqrt{1-\left(\frac{2|\omega|}{2+\epsilon}\right)^{2}}+O\left(\frac{1}{r}\right) .
\end{aligned}
$$

Similarly,

$$
\frac{2|\omega| x_{2}}{r}=1+\sqrt{1-\left(\frac{2|\omega|}{2+\epsilon}\right)^{2}}+O\left(\frac{1}{r}\right) .
$$

So, we have

$$
\frac{x_{2}}{x_{1}} \rightarrow \frac{1+\sqrt{1-\left(\frac{2|\omega|}{2+\epsilon}\right)^{2}}}{1-\sqrt{1-\left(\frac{2|\omega|}{2+\epsilon}\right)^{2}}}=b \text { (say) as }|a| \rightarrow \infty .
$$

Clearly $b>1$ and so we take $\delta<b-1$. Then we have

$$
\frac{x_{2}(|a|)}{x_{1}(|a|)}>1+\delta
$$

when $|a|$ is sufficiently large enough. Choosing $N=x_{1}(|a|)$, the conditions of Lemma 2 are satisfied. Noticing that $x_{1}(|a|)$ has the same order as $\sqrt{|a|}$, the lemma follows immediately. 
Proof of Theorem 1. The proof follows easily from Lemma 3. Assume that $f \in \mathcal{A}_{\Gamma}^{*}$ and that there exists a sequence $z_{1}, z_{2}, \ldots$ of complex numbers such that $\left|z_{j}\right| \rightarrow \infty$ and

$$
f(\alpha)=A_{j}(\text { constant }) \quad \text { for all } \alpha \in S\left(z_{j},(2+\epsilon) \sqrt{\left|z_{j}\right|}\right)
$$

for some arbitrary positive constant $\epsilon$. Applying Lemma 3 here, we get

$$
f(\alpha)=0 \text { for each } \alpha \text { with }|\alpha| \leq c_{j} \sqrt{\left|z_{j}\right|}
$$

for some positive constants $c_{j}$. Note that $\left|z_{j}\right| \rightarrow \infty(j \rightarrow \infty)$. As a consequence, we have $f \equiv 0$.

Similarly, we can prove the following assertion for completely multiplicative complex valued functions over $\Gamma^{*}$.

Theorem 2. Let $f \in \mathcal{M}_{\Gamma}^{*}$, which does not vanish anywhere. Assume that there exists a sequence $z_{1}, z_{2}, \ldots$ of complex numbers such that $\left|z_{j}\right| \rightarrow \infty$ and that

$$
f(\alpha)=A_{j}(\text { constant }) \quad \text { for all } \alpha \in S\left(z_{j},(2+\epsilon) \sqrt{\left|z_{j}\right|}\right)
$$

for some arbitrary positive constant $\epsilon$. Then $f \equiv 1$.

Corollary 1. Taking $\Gamma=\mathbb{Z}[i]$, we have Amer's result.

Corollary 2. Take $\Gamma=\mathcal{O}_{K}$, the ring of integers of imaginary quadratic field $K=\mathbb{Q}[\sqrt{d}]$, we have a more general version of Amer's result.

Remark 1. In the proof of Lemma 1 and hence in the proof of Theorem 1, we use the 'closure under multiplication' property of the system of points (domain). So we use principal configuration $\Gamma$ in place of any arbitrary lattice over $\mathbb{C}$ as the lattice may not be closed under multiplication. It would be interesting to see if the result can be proved in general for any arbitrary lattice in $\mathbb{C}$, without using the principal configuration $\Gamma$.

Acknowledgements. I am thankful to Prof. I. Kátai for suggesting the problem in case for ring of integers of imaginary quadratic fields which led to the idea for generalizing further for arithmetical functions over lattices or principal configuration in $\mathbb{C}$. I am also thankful to Prof. K. Chakraborty for his suggestions and comments during the discussions. 


\section{REFERENCES}

[1] M. Amer: On arithmetical functions over Gaussian integers having constant values in some domain, Publ. Math. Debrecen, 38/3-4, (1991), 185-188.

[2] B. N. Delone: The St. Petersburg School of Number Theory, History of Mathematics, 26, (2005).

[3] P. Erdős and I. Kátai : Non complete sums of multiplicative functions, Period. Math. Hungar., 1, 1971, no. 3, 209-212.

[4] P. Erdős and I. Kátai : On the growth of some additive functions on small intervals, Acta Math. Acad. Sci. Hungar., 33, (1979), no. 3-4, 345-359.

[5] I. Kátai : On the determination of an additive arithmetical function by its local behaviour, Colloquium Math., 20, (1969), 265-267.

[6] I. Kátai : Some remarks on arithmetical functions satisfying linear recursions in short intervals, Ann. Univ. Sci. Budapest. Etvs Sect. Math., 31, (1988), 135-139.

Harish-Chandra Research Institute, Chhatnag Road, Jhusi, AllaHABAD 211 019, INDIA., PHONE - 09651059359.

E-mail address, Jay Mehta: jaymehta@hri.res.in 